\title{
EXIT Chart Analysis of Iterative Demodulation and Decoding of MPSK Constellations with Signal Space Diversity
}

\author{
Nauman F. Kiyani and Jos H. Weber \\ Faculty of Electrical Engineering, Mathematics and Computer Science, \\ Delft University of Technology, Delft, the Netherlands. \\ Email(s): \{n.f.kiyani, j.h.weber\}@tudelft.nl
}

\begin{abstract}
Bit interleaved coded modulation (BICM) schemes have been shown to increase the diversity order. This paper studies the extension of such schemes with iterative demodulation and decoding (ID) using error correcting codes coupled with rotated MPSK signal constellations with different symbol mappings and signal space diversity (SSD). Using extrinsic information transfer (EXIT) charts, an analysis of the impact of the rotation angle on the performance of the system is presented. It is shown that for a given signal constellation, labeling, error correcting code and $E_{b} / N_{0}$-value, where conventional BICM-ID system's decoding trajectory gets stuck in a Rayleigh fading channel, SSD with a well considered choice of the rotation angle allows for the convergence of iterative demodulation and decoding. Furthermore, it is shown that the proposed system can lead to a significant performance gain over BICM-ID. The optimal choice of the rotation angle for the coded system is shown to be dependent upon the signal labeling and the error correcting code being employed. Factors (degrees of freedom) that can effect the convergence behavior of the iterative demodulation and decoding of the proposed system are identified and their effect is analyzed.
\end{abstract}

Index Terms-Channel coding, modulation, iterative demodulation and decoding, diversity, and EXIT chart analysis.

\section{INTRODUCTION}

Next generation communication systems are envisioned to operate with high data rates and at low signal-tonoise ratios (SNR). Diversity coupled with coding is an attractive option for such systems operating in severe fading conditions. In this paper, we present the use of practically sized error correcting (convolutional) codes coupled with signal space diversity (SSD) and rotated Mary phase shift keying (MPSK) constellations to achieve gain in wireless communication channels at low SNRs by using iterative demodulation and decoding.

The diversity order of a multi-dimensional signal set is the minimum number of distinct components between any two constellation points [1]. The diversity order can be increased to the minimum number of distinct bits rather than channel symbols by using bitwise interleaving.

This paper is based on " Iterative Demodulation and Decoding for Rotated MPSK Constellations with Convolutional Coding and Signal Space Diversity," by N. F. Kiyani and J. H. Weber, which appeared in the Proceedings of the 66th IEEE Vehicular Technology Conference (VTC), Baltimore, USA, Sept., 2007. (c) 2007 IEEE.
This allows a coded modulation system with moderate complexity to have large diversity order and achieve coding gain in fading environments [2]. Furthermore, in [3], [4] it was shown that iterative decoding (ID) can increase the minimum intersignal Euclidean distance of bit interleaved coded modulation (BICM), while retaining the desirable Hamming distance. SSD can provide performance improvement over fading channels by maximizing the diversity of a communication system [1]. Also, in [5] it was shown that for a block independent wireless communication link, improved performance can be obtained for a QPSK system without effecting its bandwidth efficiency, by rotating the signal constellation and separately interleaving the in-phase $(I)$ and quadrature phase $(Q)$ components. In [6], [7], this approach was used to improve the performance by combining with BICM. In [6] and [7], convolutional codes in a BICM system using coordinate interleaving were shown to have improved performance not only over conventional BICM system but also over trellis coded modulation (TCM).

This paper contributes the following. A new system model is proposed based on iterative demodulation and decoding technique using rotated multi-level modulation schemes. Convolutional codes are used as forward error correcting codes with the proposed scheme. An analysis of the effect of different signal mappings, the number of iterations and the effect of iterations on the optimum rotation angles in block Rayleigh fading channels is presented. Extrinsic information transfer (EXIT) charts are used to analyze this behavior and to illuminate the effect of optimum rotation on the convergence behavior of iterative demodulation and decoding. A symbol-to-bit demapper for multi-level modulation schemes is extended to incorporate iterative demodulation with SSD. Factors (degrees of freedom) which can effect the convergence behavior of the iterative demodulation and decoding are identified and their effect is analyzed by illustrative examples. Different signal mappings for QPSK and 8PSK constellations under various rotation angles are considered. This proposed scheme using rotated constellations is shown to outperform conventional BICM-ID.

The paper is organized as follows. Section II briefly outlines the main blocks of the system model. Iterative 
demodulation and decoding is discussed in Section III. Section IV presents the EXIT chart analysis, degrees of freedom and the performance curves of the new concatenated scheme. Lastly, in Section V conclusions are presented.

\section{SySTEM MODEL}

The block diagram of a system using iterative demodulation and decoding with SSD is shown in Figure 1. The encoded bits, which are punctured to a desirable rate, are interleaved using a pseudo-random interleaver, represented by $\pi$, and are mapped onto symbols using rotated constellations with pre-defined signal labeling. The pseudo-random interleaver is used to break the sequential fading correlation between consecutive bits that are being transmitted.

The encoded bits are mapped onto symbols using rotated constellations using pre-defined symbol labeling. The $I$ and $Q$ components of the mapped symbols are then separately interleaved and transmitted through the Rayleigh fading channel which is assumed to be block independent. $I$ and $Q$ interleaving uncorrelates the inphase and quadrature components. At the receiver, the received $I$ and $Q$ components are de-interleaved. The de-interleaved components along with the channel state information (CSI) are iteratively used by the symbol-tobit de-mapper and the decoder to generate estimates of the transmitted bits. It is important to note that the interleavers shown in the Figure 1 after the symbol mapper are symbol coordinate interleavers having smaller depth than a bit interleaver. Furthermore, since the bit interleaving already breaks the correlation due to fading, the purpose of the coordinate interleaving is to make the $I$ and $Q$ channels uncorrelated implying that the $I$ and $Q$ channel would experience independent fade. A single coordinate interleaver or a delay line introducing a delay that exceeds the coherence time of the channel would have the same effect and can be used instead of the two interleavers as shown in Figure 1.

The subsequent subsections describe the various blocks in more detail. The specific details of the iterative symbolto-bit de-mapping and decoding are further discussed in Section III.

\section{A. I and Q Interleaving of Rotated MPSK Constellations}

A conventional MPSK signal constellation is denoted by $\mathcal{S}_{M}=\left\{s_{l}=e^{2 \pi(l / M) j}: l=0,1, \ldots, M-1\right\}$, where the energy has been constrained to unity and each symbol corresponds to $m=\log _{2} M$ bits. Clockwise rotation over an angle $\theta$ leads to the constellation

$$
\mathcal{S}_{M}^{\theta}=\left\{s_{l}=e^{(2 \pi(l / M)-\theta) j}: l=0,1, \ldots, M-1\right\} .
$$

The symbol mapper can be represented by the one-to-one mapping function $\wp:\{0,1\}^{m} \rightarrow \mathcal{S}_{M}^{\theta}, s=\wp(\mathbf{b})$, where, $\mathbf{b}=\left(b_{1}, \cdots, b_{m}\right), b_{j} \in\{0,1\}$ represents the binary sequence and $s$ is chosen from the set $\mathcal{S}_{M}^{\theta}$ consisting of $M$ complex signal points. In case of $N$ symbol transmission each taken from the rotated constellation $\mathcal{S}_{M}^{\theta}$, let the sequence of $I$ components $\mathbf{x}=\left(x_{0}, x_{1}, \ldots, x_{N-1}\right)$ and the sequence of $Q$ components $\mathbf{y}=\left(y_{0}, y_{1}, \ldots, y_{N-1}\right)$ be interleaved by the $I$ interleaver $\eta$ and the $Q$ interleaver $\rho$, respectively, resulting in the sequences $\tilde{\mathbf{x}}=\eta(\mathbf{x})=$ $\left(\tilde{x}_{0}, \tilde{x}_{1}, \ldots, \tilde{x}_{N-1}\right)$ and $\tilde{\mathbf{y}}=\rho(\mathbf{y})=\left(\tilde{y}_{0}, \tilde{y}_{1}, \ldots, \tilde{y}_{N-1}\right)$. The transmitted waveform for the rotated and interleaved system is given by

$$
\begin{aligned}
s(t) & =\sum_{i=0}^{N-1} \tilde{x}_{i} p\left(t-i T_{s}\right) \cos \left(2 \pi f_{c} t\right) \\
& +\sum_{i=0}^{N-1} \tilde{y}_{i} p\left(t-i T_{s}\right) \sin \left(2 \pi f_{c} t\right) .
\end{aligned}
$$

where

$$
p(t)= \begin{cases}1, & 0 \leq t \leq T_{s} \\ 0, & \text { otherwise }\end{cases}
$$

$T_{s}$ is the symbol period and $f_{c}$ is the carrier frequency. The symbol representation and the associated bit-maps of QPSK and 8PSK constellations under consideration are given in Table. I. For QPSK only two symbol mappings are possible, i.e., Gray and Natural, whereas for 8PSK we have used Gray, set-partitioned (SP) and semi setpartitioned (SSP) mappings.

\section{B. Rayleigh Fading}

The transmission is assumed to be over a block independent Rayleigh fading channel with perfect CSI available at the receiver. The baseband input/output relations per channel use are given by

$$
\begin{aligned}
\tilde{r}_{i}^{I} & =\tilde{h}_{i} \tilde{x}_{i}+\tilde{n}_{i}^{I}, \\
\tilde{r}_{i}^{Q} & =\tilde{h}_{i} \tilde{y}_{i}+\tilde{n}_{i}^{Q},
\end{aligned}
$$

where the $\tilde{h}_{i}$ are normalized and independent Rayleigh fading factors with a probability density function $p(\alpha)=$ $2 \alpha e^{-\alpha^{2}}, \alpha>0$ and thus, $\mathcal{E}\left[\left(\tilde{h}_{i}\right)^{2}\right]=1$. The $\tilde{n}_{i}^{I}$ and $\tilde{n}_{i}^{Q}$ are independent and identically distributed (i.i.d) Gaussian random variables with zero mean and variance $N_{0} / 2$. Separate interleaving of $I$ and $Q$ components thus, results for each symbol in transmitting the $I$ component $x_{i}$ during one fade interval and the $Q$ component $y_{i}$ during another fade interval. This kind of interleaving adds diversity to the system as $x_{i}$ and $y_{i}$ experience independent fading.

\section{Symbol Detection}

The received sequences $\tilde{\mathbf{r}}^{\mathbf{I}}$ and $\tilde{\mathbf{r}}^{\mathbf{Q}}$ are de-interleaved resulting in $\mathbf{r}^{\mathbf{I}}=\eta^{-1}\left(\tilde{\mathbf{r}}^{\mathbf{I}}\right)$ and $\mathbf{r}^{\mathbf{Q}}=\rho^{-1}\left(\tilde{\mathbf{r}}^{\mathbf{Q}}\right)$. We assume that perfect CSI is available, i.e., the fading sequence $\tilde{\mathbf{h}}=$ $\left(\tilde{h}_{0}, \tilde{h}_{1}, \ldots, \tilde{h}_{N-1}\right)$ and thus the de-interleaved fading component sequences $\mathbf{h}^{\mathbf{I}}=\eta^{-1}(\tilde{\mathbf{h}})$ and $\mathbf{h}^{\mathbf{Q}}=\rho^{-1}(\tilde{\mathbf{h}})$ are known at the receiver. Based on the received symbols we are able to calculate iteratively the estimates of the transmitted bit sequence. The iterative demodulation and decoding process is described in more detail in the next section. 


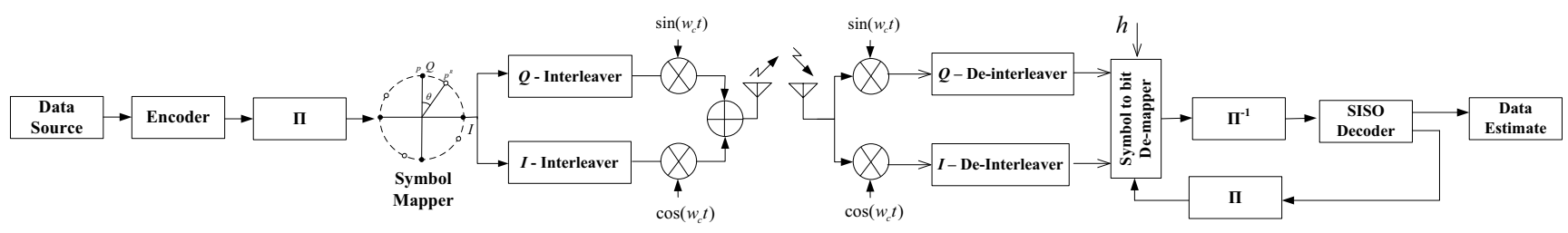

Figure 1. System Model.

TABLE I.

SYMBOL REPRESENTATIONS AND BIT-MAPS FOR UNROTATED QPSK AND 8PSK CONSTELLATIONS.

\begin{tabular}{|c|c|c|c|c|c|c|c|c|}
\hline QPSK symbols & $s_{0}=1$ & $s_{1}=j$ & $s_{2}=-1$ & $s_{3}=-j$ & & & & \\
\hline I,Q representation & $(1,0)$ & $(0,1)$ & $(-1,0)$ & $(0,-1)$ & & & & \\
\hline bit-map (Gray) & 00 & 01 & 11 & 10 & & & & \\
\hline bit-map (Natural) & 00 & 01 & 10 & 11 & & & \\
\hline \hline 8PSK symbols & $s_{0}=1$ & $s_{1}=e^{j \pi / 4}$ & $s_{2}=j$ & $s_{3}=e^{3 j \pi / 4}$ & $s_{4}=-1$ & $s_{5}=e^{5 j \pi / 4}$ & $s_{6}=-j$ & $s_{7}=e^{7 j \pi / 4}$ \\
\hline I,Q representation & $(1,0)$ & $(\sqrt{1 / 2}, \sqrt{1 / 2})$ & $(0,1)$ & $(-\sqrt{1 / 2}, \sqrt{1 / 2})$ & $(-1,0)$ & $(-\sqrt{1 / 2},-\sqrt{1 / 2})$ & $(0,-1)$ & $(\sqrt{1 / 2},-\sqrt{1 / 2})$ \\
\hline bit-map (Gray) & 000 & 001 & 011 & 010 & 110 & 111 & 101 & 100 \\
\hline bit-map (SP) & 000 & 001 & 010 & 011 & 100 & 101 & 110 & 111 \\
\hline bit-map (SSP) & 000 & 101 & 010 & 111 & 100 & 001 & 110 & 011 \\
\hline
\end{tabular}

\section{Iterative Demodulation AND Decoding}

Figure 1 shows the serial concatenation of a softinput soft-output (SISO) symbol-to-bit demodulator (indicated as "symbol-to-bit de-mapper") and a SISO decoder which are employed to iteratively demodulate and decode the received $\mathbf{r}^{\mathbf{I}}$ and $\mathbf{r}^{\mathbf{Q}}$ de-interleaved components. The receiver processes the blocks of received symbols and calculates the estimates of the transmitted data bits by the cooperative assistance of the symbol-to-bit de-mapper and the decoder. The symbol-to-bit de-mapper processes the received symbols to produce the extrinsic information which is passed to the SISO decoder. The demodulator has the CSI information available. The extrinsic information is updated by the SISO decoder and is fed back to the symbol-to-bit demapper. The receiver, thus iterates between the demodulator and the decoder to reliably estimate the transmitted bits. If the code itself does not use iterative decoding, in case of a conventional convolutional code, as shown in Figure 1, then there would be a single iteration of decoding for every iteration of demodulation. On the other hand, if the code is iteratively decoded, as it is the case for LDPC codes, then for every iteration of demodulation there are a specified number of internal iterations within the decoder before the extrinsic information is updated for feed-back. The following subsections describe the demodulation and the decoding blocks, for the cases of MPSK constellations and SISO decoding (convolutional decoding), in more detail.

\section{A. Iterative Symbol-to-Bit De-mapping for MPSK Con- stellations}

The symbol-to-bit de-mapper operates on a symbol-bysymbol basis to compute the log likelihood ratio (LLR) of the coded bits associated with each received symbol. The symbol-to-bit de-mapper, with CSI information, computes the LLR at iteration ' $q$ ' of $b_{i, j}$ which is the $j^{\text {th }}$ bit of the $i^{t h}$ received symbol where $j=1,2, \ldots, m ; i=$ $0,1, \ldots, N-1$, denoted by $\lambda^{q}\left(b_{i, j}\right)$, as in [8], which is modified to incorporate signal space diversity and feedback from the SISO decoder and is given by

$$
\lambda^{q}\left(b_{i, j}\right)=\log \frac{P_{r}\left(b_{i, j}=0 \mid r_{i}, h_{i}, \omega^{q-1}\left(b_{i, f}\right)\right)}{P_{r}\left(b_{i, j}=1 \mid r_{i}, h_{i}, \omega^{q-1}\left(b_{i, f}\right)\right)},
$$

where $\mathbf{r}=\left(r_{0}, r_{1}, \cdots, r_{N-1}\right)$ is the received sequence, $\mathbf{h}=\left(h_{0}, h_{1}, \cdots, h_{N-1}\right)$ is the fading sequence, $f=$ $1, \cdots, m$, and $\omega^{q-1}$ is the extrinsic information calculated by the SISO decoder in the previous iteration and fed-back as a priori information. The equation (4) can be rewritten and simplified as

$$
\begin{aligned}
\lambda^{q}\left(b_{i, j}\right) \approx & \max _{\mathbf{a} \in\{0,1\}^{m}: a_{j}=0}\left[E_{i}(\wp(\mathbf{a}))+\sum_{k=1, k \neq j}^{m}(-1)^{a_{k}}\right. \\
& \left.\frac{\omega^{q-1}\left(b_{i, k}\right)}{2}\right]-\max _{\mathbf{a} \in\{0,1\}^{m}: a_{j}=1}\left[E_{i}(\wp(\mathbf{a}))+\right. \\
& \left.\sum_{k=1, k \neq j}^{m}(-1)^{a_{k}} \frac{\omega^{q-1}\left(b_{i, k}\right)}{2}\right]
\end{aligned}
$$

where $\wp($.$) is the mapping function introduced in Subsec-$ tion II-A and $E_{i}(s)$ is defined as

$$
E_{i}(s)=-\frac{1}{N_{0}}\left(\left|r_{i}^{I}-h_{i}^{I} s^{I}\right|^{2}+\left|r_{i}^{Q}-h_{i}^{Q} s^{Q}\right|^{2}\right),
$$

with $s \in \mathcal{S}_{M}^{\theta}$. In the first iteration $\omega^{0}\left(b_{i, k}\right)=0 ; \forall i, k$, is assumed. On the subsequent passes (i.e., iterations $q \geq 2)$, the extrinsic information of the bits $\omega^{q-1}\left(b_{i, k}\right)$ 
is used as a priori information by the symbol-to-bit demapper. Since we have bit interleaving, we may assume that the probabilities of the bits that compose the symbol are independent [8], [9]. From (5), it is clear that when recalculating the bit metrics for one bit, we only need to use the a priori probabilities of the other bits in the same channel symbol.

\section{B. SISO Decoder}

The SISO convolutional decoder [10] is used for convolutional decoding, as shown in Figure 1, and to generate the extrinsic LLR values of the coded bits for iterative demodulation and decoding. The a priori probability of the information bits is unavailable and is not used in the entire process. The extrinsic information passed from the symbol-to-bit de-mapper is de-interleaved and used to calculate the extrinsic information of the coded bits by the SISO convolutional decoder. The regenerated bit metrics are interleaved and passed to the symbol-to-bit demapper for the next iteration between the demodulator and the decoder. The final output is the hard decision based on the extrinsic bit probability of the information bits which is also the total a posteriori probability. Punctured convolutional codes are also employed to observe the effect of the code rate on the system performance.

\section{EXIT CHART ANALYSIS \& DISCUSSION}

In the subsequent subsections an analysis of the system, the capacity, the effect of the constellation mapping and the factors (or the degrees of freedom) effecting the convergence behavior of the iterative demodulation and decoding are presented.

\section{A. Capacity}

Capacity under uniform input constraints and perfect CSI, in a flat Rayleigh fading channel that is assumed to be memoryless and with continuous-output, is given by the conditional average mutual information. With the help of the chain rule of mutual information [11], the symbolwise mutual information can be decomposed into a sum of $m$ bitwise mutual information terms. This implies that there are $m$-parallel independent channels. Let $b$ denote the binary input and $\mathbf{r}$ the channel output vector. The capacity, therefore, can be given as [2]

$$
\begin{aligned}
\hat{C} & =I(b ; \mathbf{r}) \\
& =1-\frac{1}{m} \sum_{i=1}^{m} \mathcal{E}_{b, \mathbf{r}}\left[\log _{2} \frac{\sum_{s \in \mathcal{S}_{M}^{\theta}} P_{r}(\mathbf{r} \mid s)}{\sum_{s \in S_{b}^{i}} P_{r}(\mathbf{r} \mid s)}\right],
\end{aligned}
$$

where, $S_{b}^{i}$ is the subset of all the signals in $\mathcal{S}_{M}^{\theta}$ whose corresponding bit strings have the value $b \in\{0,1\}$ in position $i$. In general, the expectation in (7) cannot be evaluated in closed form. By numerical integration using the Monte Carlo method, the capacity of the BICM system can be evaluated. Figure 2 shows the effect of the constellation mapping on the capacity of BICM. Furthermore, the figure also shows that SSD-ID can increase the capacity

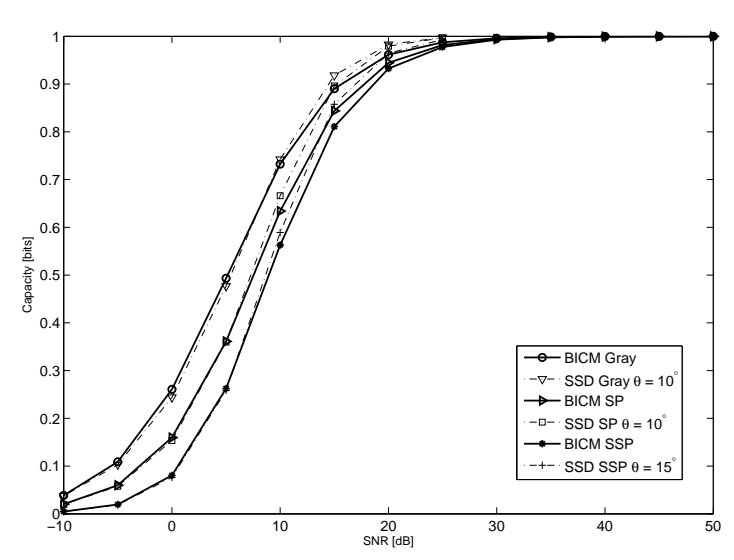

Figure 2. BICM and SSD capacity versus SNR for 8PSK over Rayleigh fading with perfect CSI using different signal constellation labeling.

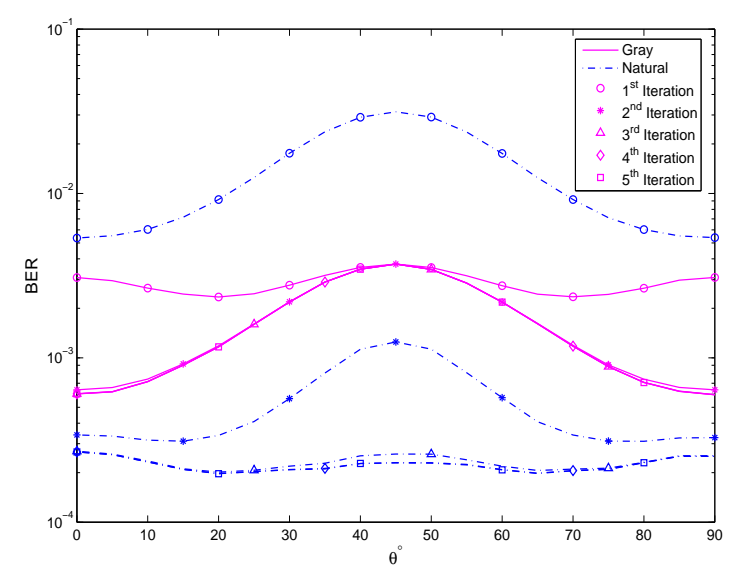

Figure 3. BER performance of QPSK SSD-ID over different rotation angles. 16-state, rate $\frac{1}{2}$ RSC codes are used with Gray and Natural signal constellation labeling in a Rayleigh fading channel at $E_{b} / N_{0}=5 \mathrm{~dB}$.

for a particular signal constellation labeling with a well considered choice of the rotation angle, as indicated by the respective dotted lines.

\section{B. Effect of Constellation Rotation}

The rotation of the signal constellation has an effect on the overall system performance of SSD-ID. The effect of the rotation of the signal constellation can be visualized by fixing the $E_{b} / N_{0}$-value and observing a system performance parameter, e.g., bit error rate (BER). A block independent Rayleigh fading channel is considered.

16 -state, rate $\frac{1}{2}$ and rate $\frac{2}{3}$ recursive systematic convolutional codes (RSC) with a generator polynomial $[37,21]_{8}$ are employed in an SSD-ID system. Each data block contains 2600 information bits. QPSK with Gray and Natural mapping, and 8PSK with Gray, SP and SSP mapping are used. Figures 3 and 4 show the BER as a function of various rotation angles for QPSK modulation scheme at $E_{b} / N_{0}=5 \mathrm{~dB}$, with code rates $\frac{1}{2}$ and $\frac{2}{3}$, respectively. The figures elucidate that the system performance, i.e., BER is dependent upon the choice of the rotation angle. The system shows best performance at $0^{\circ}$ and $20^{\circ}$ for Gray and Natural labeling, respectively, when 


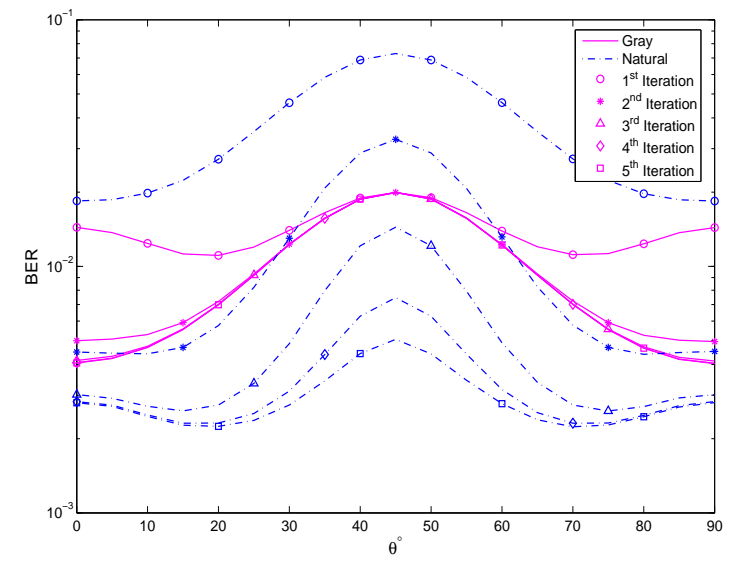

Figure 4. BER performance of QPSK SSD-ID over different rotation angles. 16 -state, rate $\frac{2}{3}$ RSC codes are used with Gray and Natural signal constellation labeling in a Rayleigh fading channel at $E_{b} / N_{0}=5 \mathrm{~dB}$.

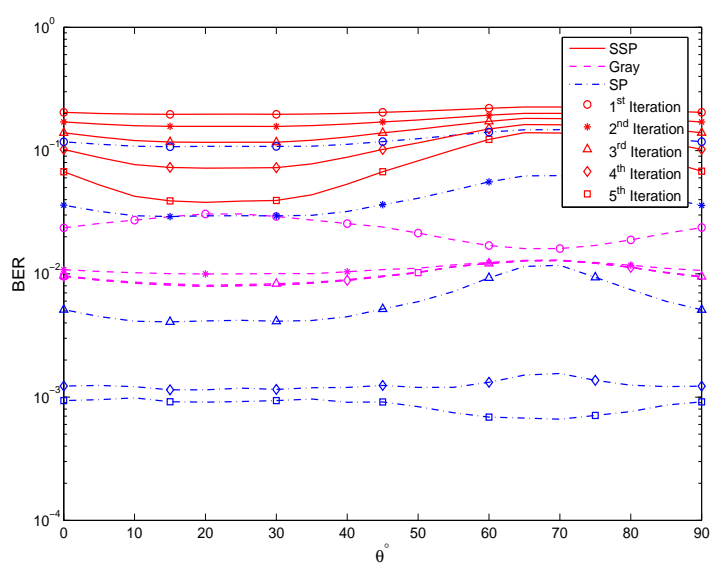

Figure 5. BER performance of 8PSK SSD-ID over different rotation angles. 16-state, rate $\frac{1}{2}$ RSC codes are used with Gray, SP and SSP signal constellation labeling in a Rayleigh fading channel at $E_{b} / N_{0}=5$ $\mathrm{dB}$.

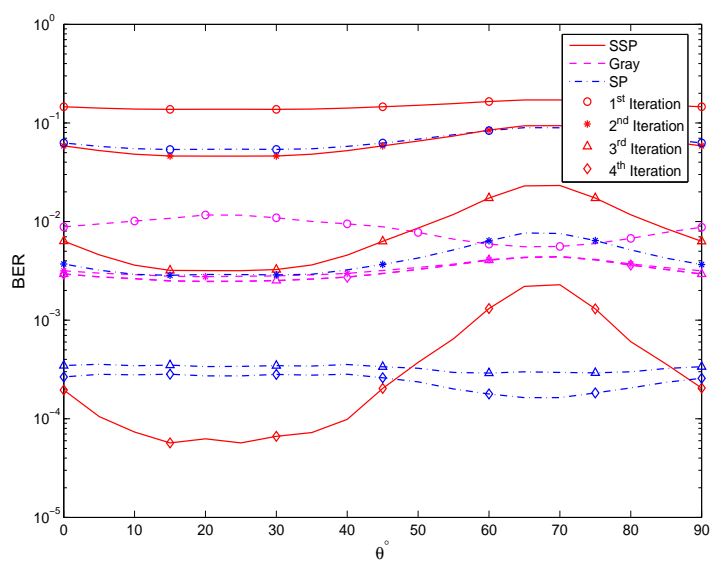

Figure 6. BER performance of 8PSK SSD-ID over different rotation angles. 16-state, rate $\frac{1}{2}$ RSC codes are used with Gray, SP and SSP signal constellation labeling in a Rayleigh fading channel at $E_{b} / N_{0}=6$ $\mathrm{dB}$.
5 iterations are performed between the demodulator and the decoder. Rotation angles having better BER performance change after the first iteration for both mappings. No further improvement in performance can be obtained with Gray labeled constellation after 2 iterations. This peculiar behavior is further discussed and elaborated upon with the help of EXIT charts. Furthermore, the rotation angles exhibiting better performance for a specific signal constellation are immune to the change in the code rate as depicted in the Figures 3 and 4. It is due to the fact that the transmitted bits are punctured to a higher rate by removing the parity bits and this lowers the error correcting capability but has no effect on the characteristic features (SSD and signal constellation rotation) of SSDID.

Similarly, Figures 5 and 6 show the BER performance of the 8PSK system as a function of the rotation angle. The figures signify the dependence of the performance of the system on the choice of the rotation angle and the signal constellation labeling. Furthermore, the figures also show that certain signal constellation labeling perform better at higher SNR region. Gray, SP and SSP labeling show best performance at $20^{\circ}, 70^{\circ}$ and $15^{\circ}$, respectively, at the $5^{\text {th }}$ iteration as in comparison to other rotational angles. At higher $E_{b} / N_{0}$-values SSP with an appropriately chosen rotation angle has better performance than Gray and SP labeling when 3 or more iterations are performed between the demodulator and the decoder, as depicted by Figure 6.

It is clear from the above figures that the system performance in terms of BER is dependent upon the signal constellation rotation, the signal constellation labeling, the SNR region of operation and the choice of the error correcting codes being employed.

\section{EXIT Chart}

The convergence behavior of the iterative demodulation and decoding can be analyzed by using mutual information to describe the flow of extrinsic information between the SISO de-mapper and the SISO decoder [12]. In EXIT charts, the exchange of extrinsic information between the symbol-to-bit de-mapper and the SISO decoder is visualized as a decoding trajectory.

In EXIT charts we predict the behavior of iterative demodulator (decoder) by solely observing its input/output relations [13]. Constituent element (demodulator/decoder) is represented by an extrinsic information transfer characteristic curve describing the relation to the input $a$ priori information to the output extrinsic information. The exchange of extrinsic information between the constituent elements is plotted as a decoding trajectory in EXIT charts. It is, therefore, imperative to separately identify the input(s) and output of the de-mapper and the decoder, respectively. For better comprehension we follow the notations in [13]. A priori input and the extrinsic output of the de-mapper is represented as $A_{1}$ and $E_{1}$, respectively. Similarly, the a priori input and the extrinsic output of the SISO decoder is represented as $A_{2}$ and $E_{2}$, respectively. 


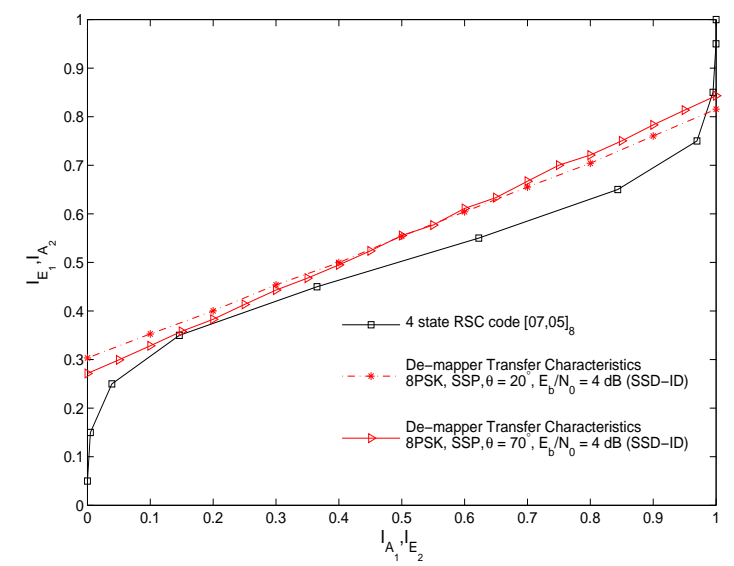

Figure 7. EXIT chart analysis of SSD-ID with 8PSK modulation and SSP labeling with $\theta=20^{\circ}, 70^{\circ}$ at $E_{b} / N_{0}=4 \mathrm{~dB}$. RSC code with generator polynomial $(07,05)_{8}$ and rate $\frac{1}{2}$ are used.

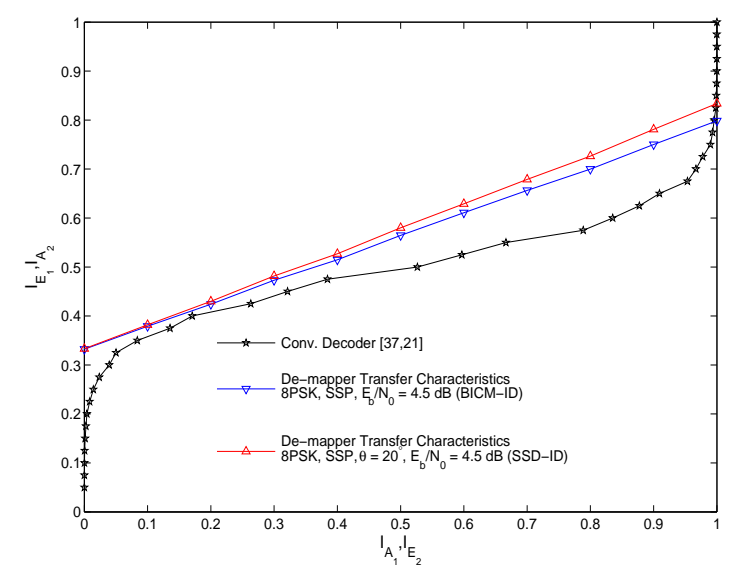

Figure 8. EXIT chart analysis of SSD-ID and BICM-ID with 8PSK modulation and SSP labeling at $E_{b} / N_{0}=4.5 \mathrm{~dB}$. RSC code with generator polynomial $(37,21)_{8}$ and rate $\frac{1}{2}$ are used.

The variables $A_{1}, E_{1}, A_{2}$ and $E_{2}$ denote the LLR-values. The iteration starts at the origin with $q=1$ and zero a priori knowledge. The iterative process stops if an intersection of both characteristic transfer curves occurs in the EXIT chart.

\section{Transfer Characteristics of Symbol-to-bit De-mapper \& SISO Decoder}

A code bit is modeled as an outcome of the binary random variable (RV) $B$. The LLR-values $\lambda$ and $\omega$ being exchanged between the demodulator and the decoder are modeled as outcomes of the RVs $E_{1}$ and $E_{2}$, respectively. The RVs are described with their conditional probability density functions (PDF). The decoding algorithm is analyzed by observing only a single parameter of these PDFs [13], i.e., the mutual information. The information transfer through the de-mapper is controlled by the amount of available a priori knowledge $A_{1}$ and by the choice of the symbol mapping. Moreover, in SSD the choice of the rotation also plays a critical role as is shown by the EXIT charts in the subsequent subsection. In [13], it has been observed that the extrinsic information LLR-values

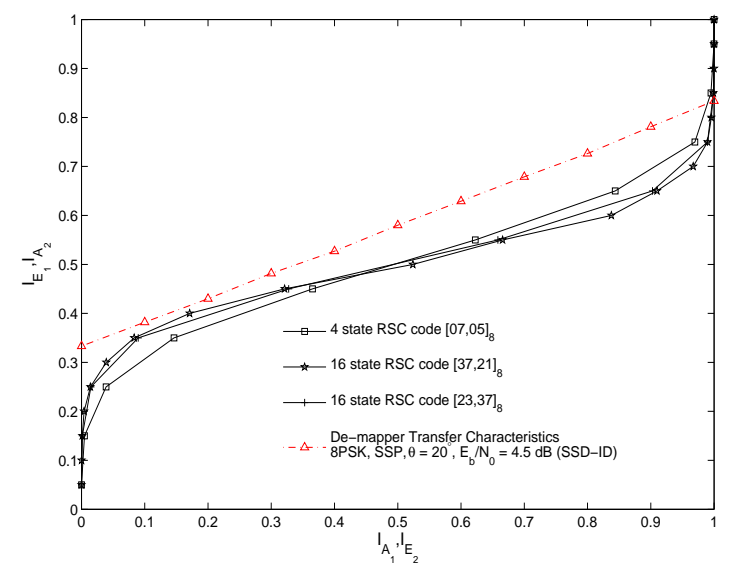

Figure 9. EXIT chart analysis of rate $\frac{1}{2}$ RSC codes with 8PSK modulation and SSP labeling, $\theta=20^{\circ}$ at $E_{b} / N_{0}=4.5 \mathrm{~dB}$.

$E_{2}$ (i.e., $A_{1}$ ), fed-back from the decoder are Gaussian distributed. Additional, it has also been observed that due to the interleaving a priori LLR-values $A_{1}$ stay uncorrelated over many iterations. Hence, the a priori input $A_{1}$ in our calculations is assumed to be Gaussian distributed. The mutual information $I_{A_{1}}=I\left(B ; A_{1}\right)$ between the transmitted bits $B$ and the LLR-values $A_{1}$ to the de-mapper can be given as

$$
\begin{aligned}
I_{A_{1}} & =I\left(A_{1} ; B\right) \\
& =\frac{1}{2} \sum_{b_{i}=0,1} \int_{-\infty}^{\infty} p_{A_{1}}\left(\xi \mid B=b_{i}\right) \\
& \cdot \quad \log _{2} \frac{2 p_{A_{1}}\left(\xi \mid B=b_{i}\right)}{p_{A_{1}}(\xi \mid B=0) p_{A_{1}}(\xi \mid B=1)} d \xi .
\end{aligned}
$$

which can be closely approximated with the time average over $N m$ coded bits [14]

$$
I_{A_{1}} \approx 1-\frac{1}{N m} \sum_{n=1}^{N m} \log _{2}\left(1+e^{(-1)^{b_{n}} \cdot \omega_{n}}\right)
$$

where $b_{n}$ is the $n^{\text {th }}$ coded bit and $w_{n}$ is the corresponding a priori LLR-value. For a given value of the input mutual information $I_{A_{1}}$, the output mutual information $I_{E_{1}}$ is calculated by Monte Carlo simulations [13]. The mutual information $I_{E_{1}}$ can be viewed as a function of $I_{A_{1}}$, $E_{b} / N_{0}$ and the rotation angle $\theta$, i.e.,

$$
I_{E_{1}}=T_{1}\left(I_{A_{1}}, E_{b} / N_{0}, \theta\right)
$$

The extrinsic transfer characteristics of a SISO decoder describe the input/output relationship between the input $A_{2}$ and the extrinsic output $E_{2}$. It is independent from $E_{b} / N_{0}$ value and can be computed by assuming $A_{2}$ to be Gaussian distributed [13]. The transfer characteristic of the SISO decoder is denoted by

$$
I_{E_{2}}=T_{2}\left(I_{A_{2}}\right)
$$




\section{E. EXIT Chart and the Convergence Behavior}

An EXIT chart analysis of SSD-ID system, as shown in Figure 1, is presented in this subsection. The purpose is to analyze the effect of the constellation rotation and SSD on the convergence behavior of the system. Illustrative examples are used to identify the degrees of freedom provided by the SSD-ID system.

Figure 7 shows as an example the performance gain of a system employing SSD-ID by the use of a proper choice of the rotation angle in a Rayleigh fading channel at $E_{b} / N_{0}=4 \mathrm{~dB}$ with 8 PSK modulation and SSP labelling. RSC code of information block length 2600 with a generator polynomial $[07,05]_{8}$ and rate $\frac{1}{2}$ is used. In Figure 7, the decoding trajectory of SSD-ID with a rotation angle $\theta=70^{\circ}$ gets stuck at low mutual information after a few iterations as the transfer characteristic curves of the decoder and de-mapper intersect, whereas a rotation angle of $\theta=20^{\circ}$ opens a narrow "tunnel". This opening of the narrow tunnel allows for the convergence of the iterative decoding towards low bit error rate (BER). This convergence towards low BER, although being slow, is possible since both the decoder and the de-mapper transfer characteristic curves do not intersect anymore. Thus SSD-ID with a proper choice of rotation angle provides an extra degree of freedom as compared to the conventional BICM-ID system.

Figure 8 depicts an additional benefit of SSD-ID as compared to the BICM-ID system, i.e., the early convergence (fewer iterations) of the iterative demodulation and decoding. This is due to the fact that for the same $E_{b} / N_{0^{-}}$ value the transfer characteristic curve of the symbol mapper for SSD-ID is always above the symbol mapper characteristic transfer curve for BICM-ID. This results in fewer iterations to achieve the same BER performance.

\section{F. Effect of different codes}

The structure of the error correcting code being employed provides another degree of freedom. In RSC codes the generator polynomials can be visualized as a selection criterion. In the context of SSD-ID these selecting criteria are the degrees of freedom to optimally choose the system parameters.

Figure 9 shows extrinsic transfer characteristics of rate $\frac{1}{2}$ RSC codes with 8 PSK signal constellation, SSP labeling, $E_{b} / N_{0}=4.5 \mathrm{~dB}$ and a rotation of $\theta=20^{\circ}$. It is interesting to see that 4 -state RSC code given by the generator polynomial $[07,05]_{8}$ has better performance at low mutual information but as the a priori information increases their performance is worse than the 16-state RSC codes.

\section{G. Results}

Figures 10 and 11 quantify the gain that is achieved by using SSD-ID in comparison to BICM-ID. A maximum of 10 iterations between the demodulator and the decoder are performed. The fading is assumed to be flat and Rayleigh distributed.

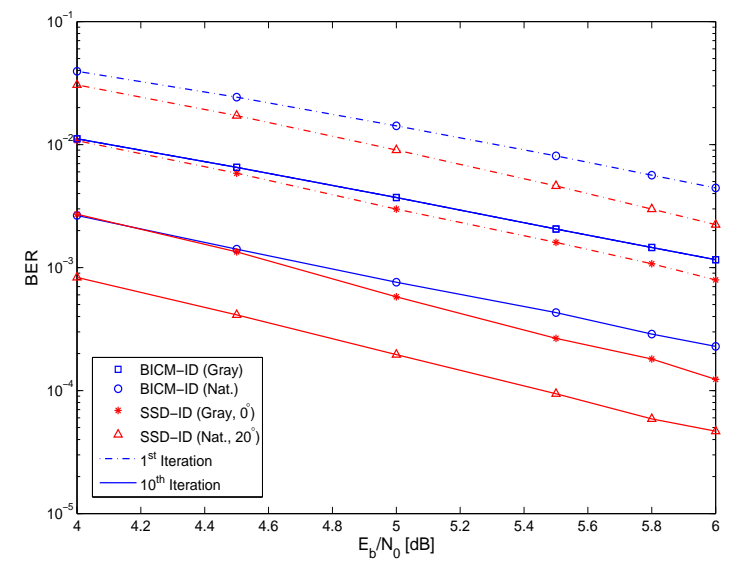

Figure 10. BER performance of SSD-ID and BICM-ID with QPSK signal constellation. Gray and Natural labeling are used. 16-state RSC codes of length 2600 and rate $\frac{1}{2}$ are employed.

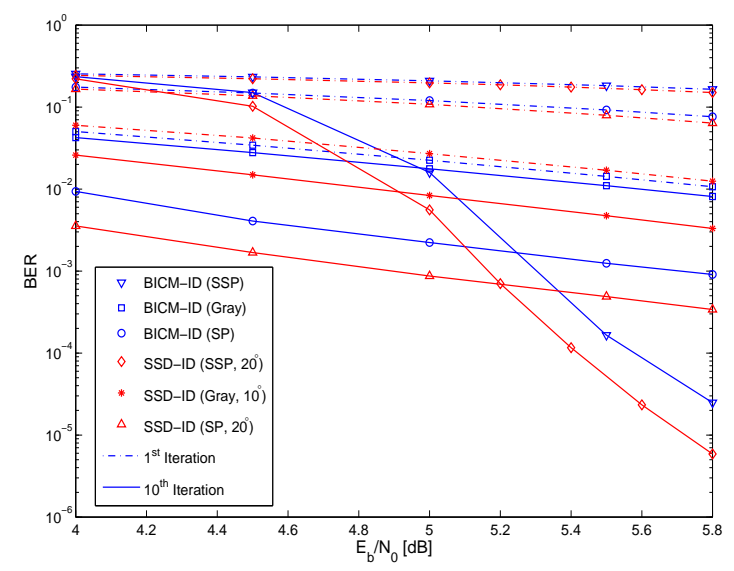

Figure 11. BER performance of SSD-ID and BICM-ID with 8PSK signal constellation. Gray, SP and SSP labeling are used. 16-state RSC codes of length 2600 and rate $\frac{1}{2}$ are employed.

Figures 10 and 11 show comparative performance gain of SSD-ID over BICM-ID by the use of 16-state RSC code with rate $\frac{1}{2}$, information block length of 2600 and generator polynomial $[37,21]_{8}$ with QPSK and 8PSK signal constellations. Figure 10 shows the performance of QPSK signal constellation with Gray and Natural mapping. Gray labeled BICM-ID does not show any performance gain after multiple iterations. SSD-ID outperforms BICM-ID over all iterations and all mappings, e.g., the Natural labeled rotated SSD-ID has a performance improvement of $1.1 \mathrm{~dB}$ at a BER of $2.3 \times 10^{-4}$ at the $10^{\text {th }}$ iteration as shown in Figure 10. Figure 11 shows the comparative performance of 8PSK signal constellation with Gray, SP and SSP labeling employed in an SSD-ID and BICM-ID system. In this figure also, SSD-ID outperforms BICM-ID. The performance after $1^{\text {st }}$ iteration is very similar but the gain provided by SSDID becomes more clear as more iterations are performed. At the $10^{\text {th }}$ iteration SSD-ID outperforms BICM-ID over all mappings, e.g., SSP labeled rotated SSD-ID has a performance improvement of $0.14 \mathrm{~dB}$ at a BER of $1 \times$ $10^{-3}$ over BICM-ID. 
The results commensurate the EXIT chart analysis presented earlier. In EXIT chart analysis it was evident that at any $E_{b} / N_{0}$-value with a well-considered choice of the rotation angle SSD-ID would have a better performance than BICM-ID. This better performance can either be considered as early convergence (fewer iterations required) to achieve a low BER value or eventual (slow with more iterations required) but possible convergence to a low BER value. Both of these criteria are possible due to the fact that the extrinsic information transfer curve of SSD-ID mapper with a well-considered choice of rotation angle is always above the BICM-ID mapper curve for a given decoder extrinsic information transfer curve.

\section{CONCLUSions}

In this paper, we have investigated the performance of SSD-ID system in Rayleigh fading channels. The performance of the proposed scheme was compared with the BICM-ID. A symbol-to-bit de-mapper for multi-level modulation schemes was extended to incorporate iterative demodulation and decoding with SSD. Using EXIT charts an analysis was presented on the effect of the rotation on the overall performance of the system. It was shown that with a well considered choice of the rotation angle the convergence of the iterative demodulation and decoding towards low BER is possible. The choice of the rotation angle was shown to be dependent upon the signal constellation labeling and the choice of the error correcting code being employed. Factors or degrees of freedom which can effect the system performance, such as, the choice of the signal constellation rotation, the signal constellation labeling, the generator polynomial for RSC codes, and the SNR region of operation, were discussed with illustrative examples. Furthermore, it was shown that SSD-ID with a well considered choice of the rotation angle and signal constellation labeling provides significant performance improvement over the conventional BICM-ID system.

\section{ACKNOWLEDGMENT}

This work was supported by STW under McAT project DTC.6438.

\section{REFERENCES}

[1] J. Boutros and E. Viterbo, "Signal space diversity: a power and bandwidth-efficient technique for the Rayleigh fading channel," IEEE Trans. Information Theory, vol. 44, no. 4, pp. 1453-1467, July 1998.

[2] G. Caire, G.Taricco, and E. Biglieri, "Bit-interleaved coded modulation," IEEE Trans. Information Theory., vol. 44, no. 3, pp. 927-945, May. 1998.

[3] X. Li and J. A. Ritcey, "Bit-interleaved coded modulation with iterative decoding and 8PSK signaling," IEEE Trans. on Comm., vol. 50, no. 8, pp. 1250-1257, Aug. 2002.

[4] A. Chindapol and J. A. Ritcey, "Design, analysis, and performance evaluation for BICM-ID with square QAM constellations in Rayleigh fading channels," IEEE Journal on Selected Areas in Comm., vol. 19, no. 5, pp. 944-957, May 2001.
[5] C. Schlegel and D. J. Costello Jr., "Bandwidth efficient coding for fading channels: Code construction and performance analysis," IEEE Journal on Selected Areas in Comm., vol. 7, no. 9, pp. 1356-1368, Dec. 1989.

[6] Z. A. Khan and B. S. Rajan, "Bit and co-ordinate interleaved coded modulation," Proc. IEEE Global Telecomm. Conf., vol. 3, pp. 1595-1599, Nov.-Dec. 2000.

[7] A. Chindapol and J. A. Ritcey, "Bit-interleaved coded modulation with signal space diversity in Rayleigh fading," Proc. 33rd Asilomar Conf. Signals, Systems, Computers., vol. 2, pp. 1003-1007, Oct. 1999.

[8] A. Stefanov and T. M. Duman, "Turbo coded modulation for systems with transmit and receive antenna diversity over block fading channels:system model, decoding approaches and practical considerations," IEEE Journal on Selected Areas in Comm., vol. 19, no. 5, pp. 958-968, May 2001.

[9] B. Lu, G. Yue, and X. Wang, "Performance analysis and design optimization of LDPC-coded MIMO OFDM systems," IEEE Trans. on Signal Process., vol. 52, no. 2, pp. 348-361, Feb. 2004.

[10] S. Benedetto, D. Divsalar, G. Montorsi, and F. Pollara, "A soft-input soft-ouput APP module for iterative decoding of concatenated codes," IEEE Comm. Letters, no. 1, pp. 22-24, Jan. 1997.

[11] T. Cover and J. Thomas, Elements of Information Theory. New York, NY: Wiley, 1991.

[12] S. ten Brink, "Convergence behavior of iteratively decoded parallel concatenated codes," IEEE Trans. on Comm., vol. 49, no. 10, pp. 1727-1737, Oct. 2001.

[13] — - "Designing iterative decoding schemes with the extrinsic information transfer chart," AEÜ Int. J. Electron. Comm., vol. 54, no. 6, pp. 389-398, Nov. 2000.

[14] M. Tüchler, "Design of serially concatenated systems depending on the block length," IEEE Trans. on Comm., vol. 52, no. 2, pp. 209-218, Feb. 2004.

Nauman F. Kiyani is currently a Ph.D. candidate at Delft University of Technology, The Netherlands. He received his B.Sc. and M.Sc. degrees in electrical engineering from Pakistan and Sweden, respectively. He was a Visiting Researcher at the Georgia Institute of Technology, Atlanta, GA, USA. His research interests include channel coding, modulation and diversity schemes.

Jos H. Weber was born in Schiedam, The Netherlands, in 1961. He received the M.Sc. (in mathematics, with honors), Ph.D., and MBT (Master of Business Telecommunications) degrees from Delft University of Technology, Delft, The Netherlands, in 1985,1989 , and 1996, respectively. He is a senior member of IEEE. Since 1985 he has been with the Electrical Engineering Department of Delft University of Technology. First he worked as a research assistant and later as an assistant professor at the Information Theory Group. Currently, he is an associate professor at the Wireless and Mobile Communications Group. He was a Visiting Researcher at the University of California, Davis, CA, USA, and at the University of Johannesburg, South Africa. His main research interests are in the area of channel and network coding. 